\title{
On mutual information, likelihood-ratios and estimation error for the additive Gaussian channel
}

\author{
Moshe Zakai*
}

May 15, 2005

\begin{abstract}
This paper considers the model of an arbitrarily distributed signal $x$ observed through an added independent white Gaussian noise $w, \quad y=x+w$. New relations between the minimal mean square error of the non-causal estimator and the likelihood ratio between $y$ and $w$ are derived. This is followed by an extended version of a recently derived relation between the mutual information $I(x ; y)$ and the minimal mean square error. These results are applied to derive infinite dimensional versions of the Fisher information and the de Bruijn identity. A comparison between the causal and non-causal estimation errors yield a restricted form of the logarithmic Sobolev inequality. The derivation of the results is based on the Malliavin calculus.
\end{abstract}

Keywords: Mutual information, Gaussian channel, minimal mean square estimation error, relative entropy, Malliavin calculus, nonlinear filtering, the logarithmic Sobolev inequality.

${ }^{*}$ Department of Electrical Engineering, Technion-Israel Institute of Technology, Haifa 32000, Israel. Email: zakai@ee.technion.ac.il 


\section{Introduction}

Let $w_{t}, 0 \leq t \leq T$ denote the standard $d$-dimensional Wiener process and $w_{t}^{\prime}$ the related white noise. The white noise channel is, roughly speaking, defined by $y^{\prime}(t)=x^{\prime}(t)+w_{t}^{\prime}, 0 \leq t \leq T$ where $x^{\prime}(t)$ is a signal process independent of the white noise process $w_{t}^{\prime}$. In the context of detection theory, the key entity is $\ell(y)$, the likelihood ratio, i.e. the Radon-Nikodym derivative of the measure induced by the $\left\{y^{\prime}(t), t \in[0, T]\right\}$ process with respect to the measure induced by the white noise $\left\{w_{t}^{\prime}, t \in[0, T]\right\}$. In the context of filtering theory the key entities are the causal and the non causal estimates, i.e. the conditional mean $E\left(x_{t}^{\prime} \mid y_{\eta}^{\prime}, 0 \leq \eta \leq t\right)$ or $E\left(x_{t}^{\prime} \mid y_{\eta}^{\prime}, 0 \leq \eta \leq T\right)$ respectively. In addition to this pair of random entities, there are also averaged entities such as the averaged minimal errors which amount to $\int_{0}^{T} E\left(x_{t}^{\prime}-E\left(x_{t}^{\prime} \mid y_{\eta}^{\prime}, \eta \in[0, t]\right)\right)^{2} d t$, or $\int_{0}^{T} E\left(x_{t}^{\prime}-E\left(x_{t}^{\prime} \mid y_{\eta}^{\prime}, \eta \in[0, T]\right)\right)^{2} d t$; and on the other hand, the mutual information between the paths $\left\{y=\left(y_{\eta}, \eta \in[0, T]\right)\right\}$ and $\left\{x=\left(x_{\eta}, \eta \in[0, T]\right)\right\}$, i.e.

$$
E \log \frac{d P(x ; y)}{d(P(x) P(y))}
$$

where the expectation is w.r. to the $P(x ; y)$ measure, and also the relative entropy $E \ell(y)$. Relations between the likelihood ratio $\ell(y)$ and the causal conditional expectation were discovered in the late 60 's and this was soon followed by a relation between the mutual information and the causal mean square error [10], [5], 9]. These relations which involved causal mean square errors were based on the Ito calculus. Similar problems for the non causal estimator were also considered [6], [8]. The formulation and results in the non causal case were restricted to the finite dimensional time discrete model of the Gaussian channel. Recently, however, Guo, Shamai and Verdú (GSV) [7] applied information theoretic arguments to derive new interesting results relating the mutual information with non causal estimation in Gaussian channels.

The Ito calculus which has proved to be a powerful tool for the relations associated with causal estimation could not be applied to problems related to non causal problems which explains the slow progress in the direction of relations for non causal estimates. However, the development of the Malliavin calculus, namely, the stochastic calculus of variation which was introduced in the mid 70's led in the early 80 's to results which prove to be a very useful tool for the non causal type of problems. 
The purpose of this paper is to apply the Malliavin calculus in order to derive the extension of the finite discrete time relations between non causal estimation and likelihood ratios to continuous time (section 4), and to prove an extended version of the results of [7] relating the mutual information with causal estimation error (section 5). The modelling of the additive Gaussian channel on the abstract Wiener space (in contrast to the $d$-dimensional Wiener process on the time interval $[0, T]$ ) yield in sections 4 and 5 results of wide applicability, e.g. for the filtering and transmission of images and random fields. The relation of these results to the de Bruijn identity, causal filtering and the logarithmic Sobolev inequality are discussed in sections 6 and 7 .

In the next section we define the Abstract Wiener Space which generalizes the classical $d$-dimensional Wiener process, and formulate the additive Gaussian channel which will be considered in the paper. Also, the problems considered in sections 4 and 5 are outlined in this section. Section 3 is a very short introduction to the Malliavin calculus. Section 4 presents the results relating likelihood ratios (R-N derivatives) with non-causal least square estimates cf. remark 2 in section 4 for possible applications of these results to nonlinear filering. In section 5 we derive an extended version of the GSV results. These results are applied in section 6 to consider the notions of Fisher information and the de Bruijn identities in an infinite dimensional setup. Section 7 deals with abstract Wiener spaces endowed with a time parameter. This enables the comparison of results for causal estimations with corresponding results for non-causal estimation. It is shown that a restricted form of the logarithmic Sobolev inequality follows directly from the results derived in this paper.

Acknowledgement: We wish to express our thanks to Shlomo Shamai for calling our attention to the problems considered in this paper and providing us with a preliminary version of [7], and to Suleyman Üstünel and Ofer Zeitouni for useful comments. 


\section{The underlying Wiener space and the additive channel model}

A. Consider, first, a standard one-dimensional Wiener process on $[0,1]$, say $w(t), t \in$ $[0,1]$. Let $\left\{\eta_{i}(t), i=1,2, \cdots, t \in[0,1]\right\}$ be a complete orthonormal base on $[0,1]$.

Set $e_{i}(t)=\int_{0}^{t} \eta_{i}(s) d s$, then $\sum_{1}^{n} \int_{0}^{1} \eta_{i}(s) d w_{s} \cdot e_{i}(t)$ converges to $w(t)$ in quadratic mean. We will denote the sequence of independent Gaussian, identically distributed (i.i.d.) random variables $\left\{\int_{0}^{t} \eta_{i}(s) d w(s)=\int_{0}^{t} \frac{d e_{i}(s)}{d t} d w(s) \quad i=1,2, \cdots\right\}$ by $\delta e_{i}, \quad i=$ $1,2, \cdots$. Then

$$
w(t)=\sum_{1}^{\infty} \delta\left(e_{i}\right) e_{i}(t) .
$$

Now $\left\{e_{i}(t), t \in[0,1], i=1,2, \cdots\right\}$ can be considered as a C.O.N. base of an Hilbert space $H$ of functions $h(t), t \in[0,1]$ with scalar product $\left(h_{1}, h_{2}\right)_{H}=\int_{0}^{1} \frac{d h_{1}(s)}{d s} \frac{d h_{2}(s)}{d s} d s$. This space $H$ is known as the Cameron Martin space. Note that the Wiener process which is continuous but not differentiable is not an element in $H$. The same notation goes over to the case of the $d$-dimensional Wiener process with $w(t), \eta(t), h(t), e(t)$ taking values in $\mathbb{R}^{d}$ and

$$
\begin{gathered}
\delta e=\int_{0}^{t} \sum_{1}^{d} \frac{d}{d t} e_{j}(s) d w_{j}(s) \\
E \delta e=0, \quad E(\delta e)^{2}=\sum_{1}^{d} \int_{0}^{1}\left(\frac{d}{d t} e_{j}(s)\right)^{2} \delta s .
\end{gathered}
$$

In this model we will consider $y(t)=x(t)+w(t)$ where $((t), t \in[0,1])$ takes values in the Cameron-Martin Space $H$ and $w(t)$, the Wiener process takes values in the space of $R^{d}$ valued continuous function considered as a Banach space $W$ under the norm $|w(t), t \in[0,1]|_{W}=\sup _{t \in[0,1]}|w(t)|_{\mathbb{R}^{d}}$.

In addition to the Banach space $W$ we have to consider the space $W^{*}$ of all continuous functionals on $W$ and it can be shown that $W^{*}$ is a dense subspace of $H$ (cf. e.g. [14]). Hence for $e \in W^{*}$, it also holds that $e \in H$ and " $e \in W^{*}$ operating on $w \in W^{\prime \prime}$ is the stochastic integral

$$
{ }_{W}\langle w, e\rangle_{W^{*}}=\delta e .
$$


An abstract model for the Wiener process in terms of the spaces $W, W^{*}, H$ and the Wiener measure $\mu_{W}$ is considered in the next subsection. The reader can skip this step by interpreting the triplet $\left(W, H, \mu_{W}\right)$ as the d-dimensional Wiener process as in equations (2.1), (2.2), (2.3).

B. The Abstract Wiener Space (AWS) is an abstraction of this model where $W$ is a separable Banach space and $H$, the Cameron-Martin space, is an Hilbert space densely and continuously embedded in $W$. The dual space to $W$ (the space of continuous linear functionals on $W$ ) is denoted $W^{*}$ and assumed to be continuously and densely embedded in $H$. The Abstract Wiener Space $\left(W, H, \mu_{W}\right)$ supports a $W$-valued random variable $w$ such that for every $e \in W^{*}, \quad \delta e:={ }_{W}\langle w, e\rangle_{W^{*}}$ is a $N\left(0,|e|_{H}^{2}\right)$ random variable. Cf. e.g. 17, or appendix B of [18] and the references therein, for further information on the AWS. Note that, unlike the classical case, the Abstract Wiener Space does not have any time-like parameter (this however can be added cf. section 7).

C. In order to introduce the general setup of the additive Gaussian channel, let $\left(W, H, \mu_{W}\right)$ be an abstract Wiener space and let $\left(H, \sigma(H), \mu_{X}\right)$ be a probability space on the Cameron-Martin Hilbert space $H$ which is induced by an $H$-valued r.v. $X$. Let $\theta=(x, w), x \in H$ and $w \in W$, set $\Theta=\{\theta\}$ and consider the combined probability space

$$
(\Theta, \mathcal{F}, \mathcal{P})=\left(\Theta, \sigma(H) \vee \sigma(W), \mu_{X} \times \mu_{W}\right)
$$

which is the space of the mutually independent 'signals' $x$ and 'noise' $w$. Now, since $H$ is continuously embedded in $W$ we can identify $x$ with its image in $W$ and defined the additive Gaussian channel as

$$
y(\theta)=\rho x+w,
$$

where $\rho$ is a free scalar 'signal to noise' parameter which will become relevant in Section 5. We will denote by $X$ and $Y$ the sigma fields induced on $W$ by the r.v.'s $x$ and $y$ respectively. Note that $y$ and $w$ are $W$ valued, $x$ is $H$ valued and we identify $x$ with its image in $W$. In fact we will make throughout this paper, just for reasons of simplicity, the additional assumption that $x$ is $W^{*}$ valued. As mentioned earlier, since $W^{*} \subset H \subset W$ we can also consider $x$ to be $H$ or $W$ valued. 
In section 4 we will be interested in the relation between two types of objects. The first class of objects is

$$
E\left((x, e)_{H} \mid Y\right) \text { and } \quad E\left(\left(x, e_{1}\right)_{H} \cdot\left(x, e_{2}\right)_{H} \mid Y\right)
$$

for $e, e_{1}, e_{2} \in W^{*}$ or globally

$$
\overline{\bar{x}}=E(x \mid Y) \quad \text { and } \quad \overline{\overline{(x, x)}}_{H}=E\left((x, x)_{H} \mid Y\right) .
$$

The second class of objects are the likelihood ratio (the $\mathrm{R}-\mathrm{N}$ derivative) between the measures induced by $y$ and the one induced by $w$ on $W$. This likelihood ratio will be denoted $\ell(w), w \in W$. Note that if $W$ is infinite dimensional then the measure induced by $x$ is singular with respect to the measure induced by $w$, (since $x \in H$ while $w \notin H)$.

In section 5 we will consider the relation between $I(X ; Y)$ or rather $d I(X ; Y) / d \rho$ and the non-causal filtering error:

$$
\left\{E|x|_{H}^{2}-|E(x \mid Y)|_{H}^{2}\right\}=E\left\{|x|_{H}^{2}-|\overline{\bar{x}}|_{H}^{2}\right\}
$$

A related result for $d(E \log \ell(w)) / d \rho$ is considered in section 6 and shown to be an extended version of the De Bruijn identity.

\section{A short introduction to the Malliavin calculus}

For further information cf. e.g. [11], 13], [17] or appendix B of [18].

(a) The gradient

Let $(W, H, \mu)$ be an AWS and let $e_{i}, i=1,2, \ldots$ be a sequence of elements in $W^{*}$. Assume that the image of $e_{i}$ in $H$ form a complete orthonormal base in $H$. Let $f\left(x_{1}, \ldots, x_{n}\right)$ be a smooth function on $\mathbb{R}^{n}$ and denote by $f_{i}^{\prime}$ the partial derivative of $f$ with respect to the $i$-th coordinate and let $\delta e$ be as discussed in the previous section.

For cylindrical smooth random variables $F(w)=f\left(\delta e_{1}, \ldots, \delta e_{n}\right)$, define $\nabla_{h} F=\left.\frac{d F(w+\varepsilon h)}{d \varepsilon}\right|_{\varepsilon=0}$. Therefore we set the following: $\nabla_{h} F=(\nabla F, h)$ where $\nabla F$, the 
gradient, is $H$-valued. For $F(w)=\delta e, \nabla F=e$, and

$$
\nabla F=\sum_{i=1}^{n} f_{i}^{\prime}\left(\delta e_{1}, \ldots, \delta e_{n}\right) \cdot e_{i}
$$

It can be shown that this definition is closable in $L^{p}(\mu)$ for any $p>1$, which means that it can be extended to a wider class of functional as we will see below. We will restrict ourselves to $p=2$, consequently the domain of the $\nabla$ operation can be extended to all functions $F(w)$ for which there exists a sequence of smooth cylindrical functions $F_{m}$ such that $F_{m} \rightarrow F$ in $L_{2}$ and $\nabla F_{m}$ is Cauchy in $L_{2}(\mu, H)$. In this case set $\nabla F$ to be the $L_{2}(\mu, H)$ limit of $\nabla F_{m}$. This class of r.v. will be denoted $\mathbb{D}_{2,1}$. It is a closed linear space under the norm

$$
\|F\|_{2,1}=E_{\mu}^{\frac{1}{2}}(F)^{2}+E_{\mu}^{\frac{1}{2}}|\nabla F|_{H}^{2} .
$$

Similarly let $K$ be an Hilbert space and $k_{1}, k_{2}, \ldots$ a complete orthonormal base in $K$. Let $\varphi$ be the smooth $K$-valued function $\varphi=\sum_{j=1}^{m} f_{j}\left(\delta e_{1}, \ldots, \delta e_{n}\right) k_{j}$ define

$$
\nabla \varphi=\sum_{j=1}^{m} \sum_{i=1}^{n}\left(f_{j}\right)_{i}^{\prime}\left(\delta e_{1}, \ldots, \delta e_{n}\right) e_{i} \otimes k_{j}
$$

and denote by $\mathbb{D}_{2,1}(K)$ the completion of $\nabla \varphi$ under the norm

$$
\|\varphi\|_{2,1}=E^{\frac{1}{2}}\left(|\varphi|_{K}^{2}+|\nabla \varphi|_{H \times K}^{2}\right) .
$$

Note that this enables us to define recursively $\nabla^{n} F(w)$ for $n>1$.

(b) The divergence (the Skorohod integral)

A few introductory remarks. Let $v(x), x \in \mathbb{R}_{n}$ take values in $\mathbb{R}_{n}, v(x)=\sum_{1} v_{i}(x) \rho_{i}$, where the $\rho_{i}$ are orthonormal vectors in $\mathbb{R}_{n}$. Assume that the $v_{i}$ and $F(x)$ are smooth and converge "quickly enough" to zero as $|x| \rightarrow \infty$. Then the following "integration by parts formula" holds

$$
\int_{\mathbb{R}_{n}}(v(x), \nabla F(x)) d x=-\int_{\mathbb{R}_{n}} F(x) \operatorname{div} v d x,
$$


where div is the divergence:

$$
\operatorname{div} v=\sum_{1}^{n} \frac{\partial v_{i}}{\partial x_{i}}
$$

Note that the gradient and divergence are differential operations, and equation (3.5) deals with integration with respect to the Lebesgue measure on $\mathbb{R}_{n}$. In this subsection we are looking for an analog of the divergence operation on $\mathbb{R}_{n}$ which will yield an integration by parts formula with respect to the Wiener measure.

Let $u(w)$ be an $H$-valued r.v. in $(W, H, \mu), u$ will be said to be in $\operatorname{dom}_{2} \delta$ if $E|u(w)|_{H}^{2}<\infty$ and there exists a r.v. say $\delta u$ such that for all smooth functionals $f\left(\delta e_{1}, \ldots, \delta e_{n}\right)$ and all $n$ the "integration by parts" relation

$$
E(\nabla f, u(w))_{H}=E(f \cdot \delta u)
$$

is satisfied. $\delta u$ is called the divergence or Skorohod integral. A necessary and sufficient condition for a square integrable $u(w)$ to be in $\operatorname{dom}_{2} \delta$ is that for some $\gamma=\gamma(u)$,

$$
\left|E(u(w), \nabla f)_{H}\right| \leq \gamma E^{\frac{1}{2}} f^{2}(w)
$$

for all smooth $f$. Note that while the definition of $\nabla f$ (at least for smooth functionals) is invariant under an absolutely continuous change of measure, this is not the case for the divergence which involves expectation in the definition. For non-random $h \in W^{*}$, $\delta h=\langle h, w\rangle$, setting $f=1$ in (3.6) yields that $E \delta h=0$. It can be shown that if $u \in \mathbb{D}_{2.1}(H)$ then $u \in \operatorname{dom}_{2} \delta$. Also, for smooth $f(w)$ it can be verified directly that

$$
\delta(f(w) h)=f(w) \delta h-(\nabla f, h)_{H}
$$

and more generally under proper restrictions

$$
\delta(f(w) u(w))=f(w) \delta u-(\nabla f, u(w))_{H} .
$$

Consequently, if $E|u|_{H}^{2}<\infty$, and $\nabla u$ is of trace class then

$$
{ }_{W^{*}}\langle u, w\rangle_{W}=\delta u+\operatorname{trace} \nabla u .
$$


where for an operator $A$ on $H$ and $e_{i}, i=1,2, \ldots$ a CONB on $H$, define

$$
\operatorname{trace} A=\sum_{1}^{\infty}\left(e_{i}, A e_{i}\right)
$$

provided the series converges absolutely and in this case $A$ is said to be of trace class. Among the interesting facts about the divergence operator, let us also note that for the classical Brownian motion and if

$$
(u(w))(\cdot)=\int_{0}^{\cdot} u_{s}^{\prime}(w) d s
$$

and $u_{s}^{\prime}(w)$ is adapted and square integrable then $\delta u$ coincides with the Ito integral i.e. $\delta u=\int_{0}^{1} u_{s}^{\prime}(w) d w_{s}$.

(c) Let $(W, H, \mu)$ be an abstract Wiener space and let $\mu_{1}$ be another probability measure on the same space $(W, \sigma\{W\})$. Assume that $\mu_{1}$ is absolutely continuous with respect to $\mu$. Set

$$
\ell(w)=\frac{d \mu_{1}}{d \mu}(w) \quad \text { and } \quad Q(w)=\{w: \ell(w)>0\}
$$

$E_{1}$ and $E_{0}$ will be used to denote the expectation with respect to the measures $\mu_{1}$ and $\mu$ respectively. We will use the convention $0 \log 0=0$ throughout the paper.

Following the definition in $3(\mathrm{~b})$, we define the divergence with respect to $\mu_{1}$ to be as follows. The $H$-valued random variable $u(w)$ will be said to be in $\operatorname{dom}_{2}^{1} \widetilde{\delta}$ if there exists a r.v., say $\widetilde{\delta} u$, which is $L^{2}$ under $u_{1}$ and such that for all smooth r.v.s $f(w)$, it holds that

$$
E_{1}(f(w) \cdot \widetilde{\delta} u)=E_{1}(u, \nabla f)_{H}
$$

The relation between $\widetilde{\delta} u$ and $\delta u$ is given by the following lemma.

Lemma 3.1 Assume that $\ell(w) \in \mathbb{D}_{2,1}, u \in \operatorname{dom}_{2} \delta, \ell \cdot \delta u \in L_{2}$ and $\ell \cdot \nabla u \in \mathbb{D}_{2,0}(H)$ and $\mu_{1} \ll \mu_{W}$ (where $\mathbb{D}_{2,0}(H)$ is the completion of (3.3) under the H-norm). Then $u \in \operatorname{dom}_{2}^{1} \widetilde{\delta}$ and

$$
\widetilde{\delta} u=\mathbf{1}_{Q}(w)\left(\delta u-(\nabla \log \ell(w), u(w))_{H}\right.
$$


Proof: Since $f(w)$ is a smooth r.v., $\ell \cdot f \cdot \delta u-f(\nabla f, u)_{H}$ is in $L_{1}$ and $\ell(w) \nabla \log \ell(w)=$ $\nabla \ell(w)$ a.s. $-\mu$. Hence

$$
\begin{aligned}
E_{1}\left(f(w) \delta u-f(w)(\nabla \log \ell(w), u)_{H}\right) & =E_{0}\left(\ell \cdot f \cdot \delta u-\ell f(\nabla \log \ell, u)_{H}\right) \\
& =E_{0}\left\{(\nabla(\ell \cdot f), u)_{H}-f(\nabla \ell, u)\right\} \\
& =E_{0}\left(\ell(\nabla f, u)_{H}\right) \\
& =E_{1}(\nabla f, u)_{H}
\end{aligned}
$$

\section{Relations between the estimation error and the likelihood ratio}

Let $(W, H, \mu),\left(H, \sigma(H), \mu_{X}\right),(\Theta, \mathcal{F}, \mathcal{P})$ and $y(\theta)=\rho x+w$ be as in section 2. We

will further assume that the $H$-valued r.v. $x$ is actually $W^{*}$ valued, and $\exp \alpha(x, h)_{H} \in$ $L^{1}\left(\mu_{X}\right)$ for all real $\alpha$ and all $h \in W^{*}$. The measures induced by $y$ and $x$ on $W$ will be denoted $\mu_{Y}$ and $\mu_{X}$ respectively. The conditional probability induced on $W$ by $y(\theta)$ conditioned on $x$ will be denoted by $\mu_{Y \mid X}$. Similarly, $\mu_{X \mid Y}$ will denote the conditional probability induced on $W^{*}$ of $x$ conditioned on $y$ (cf. e.g. [4] for the existence of these conditional probabilities).

By the Cameron-Martin theorem (cf. e.g. [18]) and since $x$ and $w$ are independent, we have

$$
\frac{d \mu_{Y \mid X}}{d \mu_{W}}(w)=\exp \left(\rho\langle w, x\rangle-\frac{\rho^{2}}{2}|x|_{H}^{2}\right), \quad w \in W
$$

which by our assumptions belongs to $L_{p}$ for all $p>0$. Hence, denoting by $\mu_{X}(d x)$ the restriction of $\mathcal{P}$ to $H$ :

$$
\begin{aligned}
\ell(w) & =\frac{d \mu_{Y}}{d \mu_{W}}(w)=\int_{H} \frac{d \mu_{Y \mid X}}{d \mu_{W}}(w, x) \mu_{X}(d x) \\
& =\int_{H} \exp \left(\rho\langle w, x\rangle-\frac{\rho^{2}}{2}|x|_{H}^{2}\right) \mu_{X}(d x)
\end{aligned}
$$


Proposition 4.1 Under these assumptions it holds that

(a)

$$
\begin{gathered}
(\nabla \ell, h)_{H}=: \nabla_{h} \ell(w)=\rho \ell(w)(\overline{\bar{x}}, h)_{H}, \quad \forall h \in H \quad \text { hence : } \\
\nabla \ell=\rho \ell(w) \overline{\bar{x}} \quad \text { or } \quad \overline{\bar{x}}=\frac{1}{\rho} \nabla \log \ell(w)
\end{gathered}
$$

a.s. $\mu_{W}$. Note that $\overline{\bar{a}}$ denotes the conditional expectation conditioned on $Y$, equation (2.7).

(b)

$$
\left(\nabla^{n} \ell(w), h_{1} \otimes \cdots \otimes h_{n}\right)_{H \otimes n}=\ell(w) \overline{\overline{\left(\prod^{n}\left(h_{i}, x\right)\right)}}
$$

(c) in particular trace $\nabla^{2} \ell(w)$ exists and a.s. $\mu_{W}$

$$
\nabla_{h_{1}, h_{2}}^{2} \ell(w)=\rho^{2} \ell(w) \overline{\overline{\left(\left(h_{1}, x\right) \cdot\left(h_{2}, x\right)\right)}}
$$

and

$$
\nabla_{h, h}^{2} \log \ell(w)=\rho^{2}\left(\overline{\overline{\left((x, h)^{2}\right)}}-(\overline{\bar{x}}, h)^{2}\right)
$$

where $\nabla_{h_{1}, h_{2}}^{2} \varphi=:\left(\nabla\left(\nabla \varphi, h_{2}\right), h_{1}\right)_{H}$, cf. also (4.8)

(d)

$$
\overline{\overline{\left(\prod_{i=1}^{n}\left(h_{i}, x\right)\right)}}=\left(h_{n}, \overline{\bar{x})} \overline{\overline{\left(\prod_{1}^{n-1}\left(h_{i}, x\right)\right)}}+\nabla_{h_{n}} \overline{\overline{\left(\prod_{i=1}^{n-1}\left(h_{i}, x\right)\right)}} .\right.
$$

Remark 1: Let $E_{1}$ denote the measure induced by $y$ on $W$ and let $E$ denote expectation w.r. to the measure in (2.5) . For an operator $A$ on $H$ and $e_{i}, i=1,2, \ldots$ a CONB on $H$, define

$$
\operatorname{trace} A=\sum_{1}^{\infty}\left(e_{i}, A e_{i}\right)
$$


provided the series converges. Consequently, we have from (4.6) and (4.3) that

$$
\begin{aligned}
E_{1} \operatorname{trace} \nabla^{2} \log \ell(w)=\rho^{2} E|x-\overline{\bar{x}}|_{H}^{2} & =\rho^{2}\left(E|x|_{H}^{2}-E|\overline{\bar{x}}|_{H}^{2}\right) \\
& =\rho^{2} E|x|_{H}^{2}-E|\nabla \log \ell(w)|_{H}^{2} .
\end{aligned}
$$

(c.f. also equations (6.3) and (6.4)).

Remark 2: (a) Consider the case where the abstract Wiener space is a classical Wiener space $\mathbb{R}^{n}$, then $u \in H$ is of the form $\int_{0}^{u} u^{\prime}(s) d s, x \in H$ is of the form $\int_{0}^{\cdot} x^{\prime}(s) d s$ where $x^{\prime}(s) \in \mathbb{R}_{n}$ and $\int_{0}^{T}\left|x^{\prime}(s)\right|_{R_{n}}^{2} d s<\infty$. Further assume that $E \int_{0}^{T}\left|x^{\prime}(s)\right|_{\mathbb{R}_{n}}^{2} d s<\infty$ and $x^{\prime}(s)$ is a.s. continuous on $[0, T]$. Then given some $t \in[0, T]$, one can consider a sequence of linear functionals $h_{n}$ such that $\left(h_{n}, x\right)$ converges in $L^{2}$ to $x(t, w)$ and extend the results of proposition 4.1] to $\overline{\bar{x}}_{t}:=E\left(x^{\prime}(t) \mid Y\right)$ for any $t \in[0, T]$. (b) Consider equation (4.2), given $w \in W$ we can replace the integration with respect to $\mu_{X}(d x)$ with a Monte-Carlo approximation. Similarly we can replace $\left\langle\nabla^{n} \ell(w), h_{1} \otimes \cdots \otimes h_{n}\right\rangle$ by applying $\nabla^{n}$ to the integrand of equation (4.2) and then replacing again the integrand with a Monte-Carlo approximation. This can then be applied to derive a numerical approximation to $\overline{\overline{\prod_{i-1}^{n}\left(h_{i}, x\right)}}$ i.e. the non-adapted non-linear fitlering of $\prod_{i=1}^{n}\left(h_{i}, x\right)$. We will not follow these directions.

The following lemma will be needed in the proof of Proposition 4.1

Lemma 4.1 Assume that $\mu_{Y}$ and $\mu_{Y \mid X}$ are absolutely continuous with respect to $\mu_{W}$ then for all bounded and measurable functions $\psi$ on $\Theta$

$$
\int_{X \times W} \psi(x, y) \frac{d \mu_{Y \mid X}}{d \mu_{W}}(y, x) \mu_{X}(d x) \times \mu_{W}(d y)=\int_{X \times W} \psi(x, y) \frac{d \mu_{Y}}{d \mu_{W}}(y) \mu_{X \mid Y}(d x ; y) \mu_{W}(d y) .
$$

\section{Proof of Lemma: Let}

$$
L=\int_{X \times Y} \psi(x, y) \mu_{X, Y}(d x, d y) .
$$


Then, by Fubini's theorem

$$
\begin{aligned}
L & =\int_{X \times Y} \psi(x, w) \mu_{Y \mid X}(d w, x) \mu_{X}(d x) \\
& =\int_{X \times Y} \psi(x, y) \frac{d_{Y \mid X}}{d \mu_{W}}(y, x) \mu_{X}(d x) \mu_{W}(d y) .
\end{aligned}
$$

Since the conditional probability $\mu_{X \mid Y}$ is regular (cf e.g. theorem 10.2.2 of [4]) we also have

$$
\begin{aligned}
L & =\int_{X \times Y} \psi(x, y) \mu_{X \mid Y}(d x, y) \mu_{Y}(d y) \\
& =\int_{X \times Y} \psi(x, y) \frac{d \mu_{Y}}{d \mu_{W}}(y) \mu_{X \mid Y}(d x, y) \mu_{W}(d y) .
\end{aligned}
$$

Proof of Proposition: From (4.1) and (4.2) and since by our assumptions we may (by dominated convergence) interchange the order of integration and differentiation

$$
\begin{aligned}
\nabla_{h} \ell(w) & =\int_{H} \rho\left(\nabla_{h}\langle w, x\rangle\right) \exp \left(\rho\langle w, x\rangle-\frac{\rho^{2}}{2}|x|_{H}^{2}\right) \mu_{X}(d x) \\
& =\int_{H} \rho(h, x) \exp \left(\rho\langle w, x\rangle-\frac{\rho^{2}}{2}|x|_{H}^{2}\right) \mu_{X}(d x) \\
& =\int_{H} \rho(h, x) \frac{d \mu_{Y \mid X}}{d \mu_{W}}(w, x) \mu_{X}(d x) .
\end{aligned}
$$

Thus, by Lemma 4.1

$$
\begin{aligned}
\nabla_{h} \ell(w) & =\int_{X} \rho(h, x) \frac{d \mu_{Y}}{d \mu_{W}}(w) \mu_{X \mid Y}(d x, w) \\
& =\rho \ell(w)(h, \overline{\bar{x}})
\end{aligned}
$$

proving (4.3). The same arguments also hold for repeated differentiation

$$
\begin{aligned}
\left(\nabla^{n} \ell(w), h_{1} \otimes \cdots \otimes h_{n}\right)_{H^{\otimes n}} & =\rho^{n} \int_{X}\left(h_{1}, x\right) \ldots\left(h_{n}, x\right) \frac{d \mu_{Y \mid X}}{d \mu_{W}}(w, x) \mu_{X}(d x) \\
& =\rho^{n} \int_{x}\left(\prod_{i=1}^{n}\left(h_{i}, x\right)\right) \frac{d \mu_{Y}}{d \mu_{W}}(w) \mu_{X \mid Y}(d x, w),
\end{aligned}
$$


which yields (4.4) (4.5) follows directly from (4.4) since

$$
\nabla_{h_{1}, h_{2}}^{2} \ell(w)=\rho^{2} \ell(w) \overline{\overline{\left(\left(h_{1}, x\right) \cdot\left(h_{2} \cdot x\right)\right)}}
$$

therefore

$$
\begin{aligned}
\nabla_{h, h}^{2} \log \ell(w) & =\frac{1}{\ell(w)} \nabla_{h, h}^{2} \ell(w)-\frac{1}{(\ell(w))^{2}}\left(\nabla_{h} \ell(w)\right)^{2} \\
& =\frac{1}{\ell(w)} \nabla_{h, h}^{2} \ell(w)-\left|\nabla_{h} \log \ell(w)\right|^{2}
\end{aligned}
$$

proving (4.5) and (4.6). From (4.4) we have

$$
\begin{aligned}
\rho^{n} \ell(w) \overline{\overline{\left(\prod_{1}^{n}\left(h_{i}, x\right)\right)}} & =\nabla_{h_{n}}\left(\nabla^{n-1} \ell(w), h_{1} \otimes \cdots \otimes h_{n-1}\right)_{H \otimes(n-1)} \\
& =\nabla_{h_{n}}\left(\ell(w) \cdot \overline{\overline{\left(\prod^{n-1}\left(h_{i}, x\right)\right)}}\right)
\end{aligned}
$$

and (4.7) follows.

We conclude this section with some results for $\delta \overline{\bar{x}}$ and $\widetilde{\delta} \overline{\bar{x}}$ (cf. part (c) of section 3). By the assumptions of this section $\overline{\bar{x}} \in \operatorname{dom}_{2} \delta$ and $\overline{\bar{x}} \in \operatorname{dom}_{2}^{1} \delta$. Therefore by (4.3)

$$
\delta \overline{\bar{x}}=\frac{1}{\rho} \delta \nabla \log \ell(w) .
$$

Note that $\mathcal{L}=\delta \nabla$ is the number operator, i.e. if $\alpha(w)$ is a square integrable r.v. of the Wiener space and $\alpha=\sum_{n=1} I_{n}$, where $I_{n}$ is the Wiener chaos decomposition of $x$; then, formally, $\mathcal{L} \alpha=\sum_{n=1} n I_{n}$. Therefore if $\mathcal{L} \alpha(w) \in L_{2}$ and $E(\mathcal{L} \alpha(w))=0$ then $\mathcal{L}^{-1} \mathcal{L} \alpha$ is well defined, consequently it holds by equation (4.3) that

$$
\ell(w)=c \cdot \exp \rho \mathcal{L}^{-1} \delta \overline{\bar{x}} .
$$

where $c$ is a normalizing constant. For $\widetilde{\delta} \overline{\bar{x}}$ we have

\section{Lemma 4.2}

$$
\widetilde{\delta} \overline{\bar{x}}=\frac{1}{\rho \ell(w)} \delta \nabla \ell(w) .
$$


Proof: By (4.3)

$$
\begin{aligned}
\delta \nabla \ell & =\rho \delta(\ell(w) \overline{\bar{x}}) \\
& =\rho \ell(w) \delta \overline{\bar{x}}-\rho(\overline{\bar{x}}, \nabla \ell)_{H} \\
& =\rho \ell(w) \delta \overline{\bar{x}}-\rho^{2} \ell(w)(\overline{\bar{x}}, \overline{\bar{x}})_{H}
\end{aligned}
$$

and

$$
\delta \overline{\bar{x}}=\frac{1}{\rho \ell(w)} \delta \nabla \ell(w)+\rho(\overline{\bar{x}}, \overline{\bar{x}})_{H} .
$$

Hence by Lemma 3.1

$$
\begin{aligned}
\widetilde{\delta} \overline{\bar{x}} & =\delta \overline{\bar{x}}-(\nabla \log \ell(w), \overline{\bar{x}})_{H} \\
& =\delta \overline{\bar{x}}-\rho|\overline{\bar{x}}|_{H}^{2} \\
& =\frac{\mathcal{L} \ell(w)}{\rho \ell(w)}
\end{aligned}
$$

\section{The GSV relation between the mutual informa- tion and the mean square of the estimation error}

Consider the setup and assumptions in the first paragraph of section 4 . The mutual information between $x$ and $y$ is defined as

$$
I(X ; Y)=\int_{X \times W} \log \frac{d \mu_{X ; Y}}{d\left(\mu_{X} \times \mu_{Y}\right)}(x, y) \mu_{X, Y}(d x, d y) .
$$

$E$ will denote expectation w.r. to the measure in (2.5), (cf. e.g. [16]). $E_{0}$ will denote expectation w.r. to the Wiener measure and $E_{1}$ will denote expectation w.r. to the measure on $W$ induced by $y$ (hence $\left.E f(y)=E_{1} f(w)=E_{0} \ell(w) f(w)\right)$.

Proposition 5.1 Under the assumptions of the previous section, it holds that

$$
\begin{aligned}
\frac{d I(X ; Y)}{d \rho} & =\rho E\left(|x|_{H}^{2}-|\overline{\bar{x}}|_{H}^{2}\right) \\
& =\rho E|x-\overline{\bar{x}}|_{H}^{2}
\end{aligned}
$$


Proof: By our assumptions, and since $\frac{d \mu_{X, Y}}{d\left(\mu_{X} \times \mu_{Y}\right)}=\frac{d \mu_{Y \mid X}}{d \mu_{W}} \cdot \frac{d \mu_{W}}{d \mu_{Y}}$, we have

$$
\begin{aligned}
I(X ; Y) & =\int_{X \times W}\left\{\log \frac{d \mu_{Y \mid X}}{d \mu_{W}}(x, y)-\log \frac{d \mu_{Y}}{d \mu_{W}}(y)\right\} \mu(d x, d y) \\
& =E\left(\rho\langle y, x\rangle-\frac{\rho^{2}}{2}|x|_{H}^{2}\right)-E \log \ell(w) .
\end{aligned}
$$

Note that $E \rho\langle y, x\rangle=\rho^{2} E|x|_{H}^{2}$, hence

$$
I(X ; Y)=\frac{\rho^{2}}{2} E|x|_{H}^{2}-E_{1} \log \ell(w)
$$

and

$$
\begin{aligned}
\frac{d I(X ; Y)}{d \rho} & =\rho E|x|_{H}^{2}-\frac{d}{d \rho} E_{0} \ell(w) \log \ell(w) \\
& =\rho E|x|_{H}^{2}-E_{0}\left(\frac{d \ell(w)}{d \rho} \cdot \log \ell(w)\right)-0 .
\end{aligned}
$$

Now,

$$
\frac{d \ell(w)}{d \rho}=\int_{X}\left(\langle x, w\rangle-\rho|x|_{H}^{2}\right) \frac{d \mu_{Y \mid X}(x)}{d \mu_{W}}(w) \mu_{X}(d x) .
$$

By lemma 4.1

$$
\begin{aligned}
\frac{d \ell(w)}{d \rho} & =\int_{X}\left(\langle x, w\rangle-\rho|x|_{H}^{2}\right) \frac{d \mu_{Y}}{d \mu_{W}}(w) \mu_{X \mid Y}(d x) \\
& =\left(\langle\overline{\bar{x}}, w\rangle-\rho \overline{\overline{\left(|x|_{H}^{2}\right)}}\right) \ell(w) .
\end{aligned}
$$

Substituting in (5.4) yields

$$
\frac{d I}{d \rho}=\rho E|x|_{H}^{2}-E_{0}\left\{\left(\langle\overline{\bar{x}}, w\rangle-\rho \overline{\overline{\left(|x|_{H}^{2}\right)}}\right) \ell(w) \log \ell(w)\right\} .
$$


Now, by (4.3)

$$
\begin{aligned}
E_{0} \ell \log \ell\langle\overline{\bar{x}}, w\rangle= & E_{0}\left(\frac{1}{\rho} \log \ell\langle\nabla \ell, w\rangle\right) \\
\text { by } \stackrel{\text { [3.8] }}{=} & E_{0} \frac{1}{\rho} \log \ell\left(\delta \nabla \ell+\operatorname{trace} \nabla^{2} \ell\right) \\
\text { by } \stackrel{\text { [3.7 }}{=} & E_{0} \frac{1}{\rho} \delta(\log \ell \nabla \ell)-E_{0} \frac{1}{\rho}(\nabla \ell, \nabla \log \ell)+E_{0} \frac{1}{\rho}\left(\log \ell \operatorname{trace} \nabla^{2} \ell\right) \\
\text { by [3.6) and [4.9] } & 0-\frac{1}{\rho} E_{0} \frac{1}{\ell}(\nabla \ell, \nabla \ell)+E_{0} \rho\left(\ell \log \ell \overline{\overline{\left(|x|_{H}^{2}\right)}}\right) \\
= & \rho E_{0} \ell(w)(\overline{\bar{x}}, \overline{\bar{x}})+E_{0} \rho \ell(w) \log \ell(w) \overline{\overline{\left(|x|_{H}^{2}\right)}} .
\end{aligned}
$$

Substituting into (5.5) yields

$$
\frac{d I}{d \rho}=\rho E|x|_{H}^{2}-\rho E|\overline{\bar{x}}|_{H}^{2}+E_{0} \rho \ell \log \ell \overline{\overline{\left(|x|_{H}^{2}\right)}}-E_{0} \rho \ell \log \ell \overline{\overline{\left(|x|_{H}^{2}\right)}}
$$

Remark (a): $E_{1} \log \ell(w)(=E \log \ell(y))$ is the relative entropy (or $I$-divergence or Kullback-Leibler number) of $\mu_{Y}$ with respect to $\mu_{W}$ (cf. e.g. [16] or [2]). Equation (5.2) relates this relative entropy to the mutual information $I(x ; y)$ for the additive Gaussian channel. By equations (5.3) and (5.1)

$$
\frac{d}{d \rho} E_{1} \log \ell(w)=\rho E_{1}|\overline{\bar{x}}|_{H}^{2}
$$

Remark (b): Consider the following generalizations to the additive Gaussian channel. Let $M$ be "the space of messages which generate the signals" $x$, i.e. $\left(M, \mathcal{B}_{\mu}, \mathcal{P}_{\mu}\right)$ is a probability space and $x=g(m), \quad m \in M$, where $g$ is a measurable from $\left(M, \mathcal{B}_{\mu}\right)$ to $H$. Then obviously $I(M, Y)=I(X ; Y)$. More generally, consider the case where $x$ and $m$ are related by some joint probability on $M \times H$ and $w$ and $m$ are conditionally independent conditioned on $x$. The extension of proposition 5.1 in this context follows along the same arguments as in theorem 13 of [7] and therefore omitted. 


\section{An extended version of the De Bruijn identity}

The Fisher information matrix $J$ associated with a smooth probability density $p\left(y_{1}, \ldots, y_{n}\right), y \in \mathbb{R}_{n}$ is defined as

$$
J=\left(\frac{\partial^{2} \log p\left(y_{1}, \ldots, y_{n}\right)}{\partial y_{i} \partial y_{j}}\right)_{1 \leq i, j \leq n}
$$

and then the Fisher information which is defined by the r.h.s. of (6.1) satisfies:

$$
E \text { trace } J=-E\left\{|\nabla \log p|_{\mathbb{R}^{n}}^{2}\right\}
$$

where $E$ is the expectation with respect to the $p$ density. The De Bruijn identity (cf. 3] or [7] and the references therein) deals with the case where $y=x+\sqrt{t} w$ where $w=w_{1}, w_{2}, \ldots, w_{n}$ and the $w_{j}, \quad j=1, \ldots, n$ are i.i.d. $N(0,1)$ and $x$ is an $\mathbb{R}_{n}$ random variable independent of $w$. It states that

$$
\frac{d}{d t} E \log p(y)=\frac{1}{2} E\left\{|\nabla \log p(y)|_{\mathbb{R}^{n}}^{2}\right\}
$$

The Fisher information matrix cannot be extended directly to the case where $y$ is infinite dimensional. However, the results of sections 4 and 5 yield some similar relations. Under the assumptions of section 5, comparing (15.1) with (5.3) we have

$$
\begin{aligned}
\frac{d}{d \rho} E_{1} \log \ell(w) & =\rho E_{1}|\overline{\bar{x}}|_{H}^{2} \\
& =\frac{1}{\rho} E_{1}|\nabla \log \ell(w)|_{H}^{2},
\end{aligned}
$$

which is "similar" to (6.2) and may be considered an extended De Bruijn identity. Note that $E_{1} \log \ell(w)$ is the relative entropy of $\mu_{Y}$ relative to $\mu_{W}$, also, note the difference between the $\rho$ and the $t$ parametrizations.

Comparing (5.1) with (4.8) yields

$$
\begin{aligned}
E_{1} \operatorname{trace} \nabla^{2} \log \ell(w) & =\rho \frac{d I(x ; y)}{d \rho} \\
& =\rho^{2} E|x|_{H}^{2}-E_{1}|\nabla \log \ell(w)|_{H}^{2},
\end{aligned}
$$


which is different from (6.1) by the $\rho^{2} E|x|_{H}^{2}$ term. Note that the validity of (6.4) is restricted to the case where $\ell(w)$ is induced by a signal plus independent noise model and not for any $\ell(w)$ which is a negative r.v. and whose expectation is 1 , cf. the concluding lines of the next section.

\section{Adding a "time parameter" to the abstract Wiener space}

Given an abstract Wiener space $(W, H, \mu)$ we can introduce the notion of continuous time on this space as follows. Let $\left\{\pi_{\theta}, 0 \leq \theta \leq 1\right\}$ be a continuous, strictly increasing, resolution of the identity on $H$ with $\pi_{0}=0, \pi_{1}=I$. Set $\mathcal{F}_{\theta}=\sigma\left\{\delta \pi_{\theta} h, h \in H\right\}$ and $\mathcal{F}$. will denote the filtration induced by $\mathcal{F}_{\theta}$ on $[0,1]$. An $H$-valued r.v. $u(w)$ will be said to be adapted to $\mathcal{F}$. if $\left(u, \pi_{\theta}, h\right)_{H}$ is $\mathcal{F}_{\theta}$ measurable for all $h \in H$ and every $\theta \in[0,1]$, (c.f. section 2.6 of [18] for more details). Let $\mathbb{D}_{2}(H)$ denote the class of $H$-valued $u(w)$

such that $E_{1}|u|_{H}^{2}<\infty$. The class of adapted square integrable random variables is a closed subspace of $\mathbb{D}_{2}(H)$ and will be denoted by $\mathbb{D}_{2}^{a}(H)$. We will denote by $\widehat{u}$ the projection of $u \in \mathbb{D}_{2}(H)$ on $\mathbb{D}_{2}^{a}(H)$, i.e.

$$
E_{1}|u-\widehat{u}|_{H}^{2}=\inf _{v \in \mathbb{D}_{2}^{a}(H)} E_{1}|u-v|_{H}^{2}
$$

(this corresponds to the dual predictable projection in martingale theory). Since $x$ is independent of $w$, and since $\overline{\bar{x}}=E_{1}(x \mid \sigma(y))$, then

$$
\widehat{x}=(\overline{\bar{x}})^{\widehat{x}}
$$

i.e. if $x$ is not measurable on the $\sigma$-field induced by $y$, project, first, $x$ on the $\sigma$-field generated by $y$ and then project on $\mathbb{D}_{2}^{a}(H)$, which is the same as replacing $u$ with $x$ in (7.1). Then (cf. e.g. [18)

$$
\ell(y)=\exp \left(\rho \delta \widehat{x}-\frac{\rho^{2}}{2}|\widehat{x}|_{H}^{2}\right) .
$$

By the same arguments as in [5] or [9] and by the assumptions of proposition 5.1]

$$
I(X ; Y)=\frac{\rho^{2}}{2} E|x-\widehat{x}|_{H}^{2} .
$$


Remarks: (a) The left hand side of (7.4) is independent of the choice of $\pi$. while $\widehat{x}$ does depend on the choice of $\pi$.. Consequently, by (17.3),$E|x-\widehat{x}|_{H}^{2}$ is independent of the particular choice of $\pi$.. (b) The validity of (7.3) and (7.4) is not restricted to the case where $x$ is independent of $w$ (cf. [9] and [18]).

By equations (5.2) and (7.4),

$$
\begin{aligned}
E_{1} \log \ell(w) & =\frac{\rho^{2}}{2} E\left(|x|_{H}^{2}-\frac{1}{2}|x-\widehat{x}|_{H}^{2}\right) \\
& =\frac{\rho^{2}}{2} E\left(-|\widehat{x}|_{H}^{2}+2(x, \widehat{x})_{H}\right)
\end{aligned}
$$

Hence

$$
E_{1} \log \ell(w)=\frac{\rho^{2}}{2} E|\widehat{x}|_{H}^{2}
$$

We conclude the paper with the following remark. Obviously

$$
E|\widehat{x}|_{H}^{2} \leq E|\overline{\bar{x}}|_{H}^{2}
$$

Hence by (7.5) and (6.3),

$$
E_{1} \log \ell(w) \leq \frac{1}{2} E_{1}|\nabla \log \ell(w)|_{H}^{2}
$$

or

$$
E_{0} \ell(w) \log \ell(w) \leq \frac{1}{2} E_{0} \ell(w)|\nabla \log \ell(w)|_{H}^{2}
$$

Setting $f^{2}(w)=c \ell(w), c>0$ then

$$
E_{0} f^{2}(w) \log |f(w)| \leq E_{0} f^{2} \cdot \log E_{0}^{\frac{1}{2}} f^{2}+E_{0}|\nabla \log f|_{H}^{2}
$$

which is the logarithmic Sobolev inequality of L. Gross on Wiener space (cf. e.g. section 9.2 of [20] and the references therein). Note, however, that (7.8) is not the complete logarithmic Sobolev inequality since as derived above, it holds only the for the case where $\ell(w)$ is the likelihood ratio associated with $x+w$ where $x$ and $w$ are independent (and not for any nonnegative $\ell(w)$ for which $E \ell(w)=1$ cf. [15], 21]).

Inequality (7.8) follows from the obvious inequality (17.6) and the equalities derived earlier in this paper. The question arises whether a similar argument can yield (7.8) 
without the restriction on $\ell(w)$ to be generated by a signal plus independent white noise. This seems to be a delicate problem; the left hand side of (17.7) can be shown to be equal to the left hand side of (7.6) without the restriction that the signal and noise are independent ([9]). However it is not clear if the right hand side of (7.7) and (17.6) are equal. 


\section{References}

[1] E.A. Carlen, "Superadditivity of Fisher's information and logarithmic Sobolev inequalities", J. Funct. Anal, vol. 101, pp. 194-211, 1991.

[2] I. Csiszar and F. Matus, "Information projections revisited," IEEE Trans. Inform. Theory, vol. 49, no. 6, pp. 1474-1490, 2003.

[3] A. Dembo, M. Cover and J.A. Thomas, "Information theoretic inequalities," IEEE Trans. Inform. Theory, vol. 37, pp. 1501-1518, 1991.

[4] R.M. Dudley, Real Analysis and Probability, Wadsworth and Brooks/Cole, 1989.

[5] T.E. Duncan, "On the calculation of mutual information," SIAM Journal of Applied Mathematics, vol. 19, pp. 215-220, July 1970.

[6] R. Esposito, "On a relation between detection and estimation in decision theory," Inform. Contr., vol. 12, pp. 116-120, 1968.

[7] D. Guo, S. Shamai, and S. Verdú, "Mutual information and minimum meansquare error in estimation, filtering and smoothing," IEEE Trans. Inform. Theory, 2005 to appear.

[8] C.P. Hatsell and L.W. Nolte, "Some geometric properties of the likelihood ratio," IEEE Trans. Inform. Theory (Corresp), pp. 616-618, vol. IT-17, Sept. 1971.

[9] T.T. Kadota, M. Zakai and J. Ziv, "Mutual information of the white Gaussian channel with and without feedback", IEEE Trans. Inform. Theory, vol. IT-17, pp. 368-371, 1971.

[10] T. Kailath, "A note on least squares estimates from likelihood ratios," Inform. Contro., vol. 13, pp. 534-540, 1968.

[11] P. Malliavin, Stochastic Analysis, Springer-Verlag, Berlin/New York, 1997.

[12] E. Mayer-Wolf and M. Zakai, "On a formula relating the Shannon information to the Fisher information for the filtering problem," in Lecture Notes in Control and Information Sciences, vol. 61, pp. 164-171. Springer-Verlag, New York, 1983.

[13] D. Nualart, The Malliavin Calculus and Related Topics, Springer Verlag, 1995. 
[14] D. Nualart and M. Zakai, "Multiple Wiener-Ito integrals possessing a continuous extension," Pr. Th. Rel. Fields, vol. 85, pp. 131-145, 1990.

[15] D. Nualart and M. Zakai, "Positive and strongly positive Wiener functionals", Barcelona Seminar on Stochastic Analysis (St. Feliu de Guixols, 1991), 132-146, Progr. Probab., 32, Birkhauser, Basel, 1993.

[16] M.S. Pinsker, Information and Information Stability of Random Variables and Processes, Holden-Day, 1964.

[17] A.S. Üstünel, "An introduction to analysis on Wiener space," Lecture Notes in Math., vol. 1610, Springer 1996.

[18] A.S. Üstünel and M. Zakai, Transformation of Measure on Wiener Space, Springer-Verlag, New York/Berlin, 1999.

[19] A.S. Üstünel and M. Zakai, "Embedding the abstract Wiener space in a probability space," J. Func. Anal., vol. 171, pp. 124-138, 2000.

[20] C. Villani, "Topics in optimal transportation," American Mathematical Society, Graduate Studies in Math. Vol. 58, 2003.

[21] E. Wong and M. Zakai, "A characterization of kernels associated with the multiple integral representation of some functionals of the Wiener process", Systems and Control Lett., vol. 2, pp. 94-98, 1982/3. 